\title{
ON THE SPACE OF PIECEWISE LINEAR HOMEOMORPHISMS OF A MANIFOLD
}

\author{
ROSS GEOGHEGAN ${ }^{1}$ AND WILLIAM E. HAVER
}

\begin{abstract}
Let $M^{n}$ be a compact PL manifold, $n \neq 4$; if $n=5$, suppose $\partial M$ is empty. Let $H(M)$ be the space of homeomorphisms on $M$ and $H^{*}(M)$ the elements of $H(M)$ which are isotopic to PL homeomorphisms. It is shown that the space of PL homeomorphisms, PLH(M), has the finite dimensional compact absorption property in $H^{*}(M)$ and hence that $\left(H^{*}(M), P L H(M)\right)$ is an $\left(l_{2}, l_{2}^{f}\right)$-manifold pair if and only if $H(M)$ is an $l_{2^{-}}$ manifold. In particular, if $M^{2}$ is a 2-manifold, $\left(H\left(M^{2}\right), P L H\left(M^{2}\right)\right)$ is an $\left(l_{2}, l_{2}\right)$-manifold pair.
\end{abstract}

1. Introduction. Let $M$ be a compact piecewise linear (PL) manifold, possibly with boundary. We shall study the pair $(H(M), P L H(M))$, where $H(M)$ denotes the space of all homeomorphisms of $M$ onto itself and $P L H(M)$ the subspace of all piecewise linear homeomorphisms. All function spaces will be assumed to have the compact-open topology.

For some years now there has been considerable interest in the question of whether $H(M)$ is an $l_{2}$-manifold; i.e., a separable metric space which is locally homeomorphic to $l_{2}$, the hilbert space of square-summable sequences. For an arbitrary compact manifold $M$, it is known that $H(M)$ is uniformly locally contractible (Chernavskii [6] and Edwards and Kirby [8]) and that $H(M) \times l_{2}$ is homeomorphic to $H(M)$ (Geoghegan [11]). By a theorem of Torunczyk [20], $H(M)$ is an $l_{2}$-manifold if and only if $H(M)$ is an ANR. When $M$ is a 2manifold, Luke and Mason [18] have shown that $H(M)$ is an ANR (hence an $l_{2}$-manifold). But it is still unknown whether or not $H\left(M^{n}\right)$ is an ANR when $n>2$.

In the PL case more is known. $P L H(M)$ is the countable union of finitedimensional compacta (Geoghegan [12]), and is uniformly locally contractible (Edwards, see [13] and Gauld [10]). Haver [13] has shown that any such space is an ANR. Torunczyk [20] has shown that any such ANR becomes an lfmanifold when multiplied by $l_{2}^{f}$ (where $l_{2}^{f}$ denotes the subspace of $l_{2}$ consisting of those sequences having only finitely many nonzero entries). Hence $P L H(M) \times l_{2}^{f}$ is an $l f$-manifold. Finally, Keesling and Wilson [16] have shown that $P L H(M) \times l_{2}$ is homeomorphic to $P L H(M)$. Hence $P L H(M)$ is an $l f-$ manifold.

In general it is not true that $P L H(M)$ is dense in $H(M)$. For example, Kirby and Siebenmann (see [17]) have shown that $H\left(S^{2} \times S^{3}\right)$ has a component containing no PL homeomorphism. They have also shown that if the

Received by the editors November 4, 1974.

AMS (MOS) subject classifications (1970). Primary 58D05, 57C99; Secondary 57E05, 58B05.

${ }^{1}$ Supported in part by NSF Grant P038761. 
third cohomology group of $M^{n}$ with $\mathbf{Z}_{2}$-coefficients is trivial, and if $n \geq 6$, or $n=5$ and $\partial M$ is empty, $P L H\left(M^{n}\right)$ is dense in $H\left(M^{n}\right)$. In low dimensions, $P L H\left(M^{n}\right)$ is always dense in $H\left(M^{n}\right)$ : this is obvious when $n=1$; see Rado [19] when $n=2$; see Bing [4] when $n=3$.

For our purposes we can avoid reference to the difficult work of Kirby and Siebenmann. Using earlier work of Connell [7] we can prove

THEOREM 1. Let $M^{n}$ be a compact PL manifold, $n \neq 4$; if $n=5$ suppose $\partial M$ is empty. Then the closure of $P L H(M)$ in $H(M)$ is the union of components of $H(M)$.

Let $H^{*}(M)$ be the subset of $H(M)$ consisting of those homeomorphisms which are isotopic to PL homeomorphisms. The above remarks show that $H^{*}(M)$ is of ten equal to $H(M)$. Theorem 1 says that [with certain dimension restrictions] $P L H\left(M^{n}\right)$ is always dense in $H^{*}\left(M^{n}\right)$.

A pair of spaces $\left(X, X^{\prime}\right)$ is an $\left(l_{2}, l_{2}\right)$-manifold pair if $X$ is an $l_{2}$-manifold, and if there exist an open cover $\mathcal{Q}$ of $X$ and open embeddings $\left\{f_{U}: U \rightarrow l_{2} \mid U\right.$ $\in \mathscr{Q}\}$ such that for each $U, f_{U}\left(U \cap X^{\prime}\right)=f_{U}(U) \cap l f$. In other words $X^{\prime}$ sits in $X$, locally, as $l_{2}$ sits in $l_{2}$.

TheORem 2. Let $M^{n}$ be a compact PL manifold, $n \neq 4$; if $n=5$, suppose $\partial M$ is empty. Then $\left(H^{*}\left(M^{n}\right), P L H\left(M^{n}\right)\right)$ is an $\left(l_{2}, l_{2}\right)$-manifold pair if and only if $H(M)$ is an $l_{2}$-manifold.

Theorem 2 is proved by showing that $P L H\left(M^{n}\right)$ has the "finite-dimensional compact absorption property" in $H^{*}\left(M^{n}\right)$ : see $\$ 3$ for the definition. This theorem, when combined with our opening remarks, yields

Corollary 1. If $M^{2}$ is a compact PL 2-manifold, $\left(H\left(M^{2}\right), P L H\left(M^{2}\right)\right)$ is an $\left(l_{2}, l_{2}\right)$-manifold pair.

Theorem 1 combined with the fact that each of $P L H(M)$ and $H(M)$ is uniformly locally contractible immediately gives:

Corollary 2. Let $M^{n}$ be as in Theorem 2. Then the inclusion PLH $\left(M^{n}\right)$ $\rightarrow H^{*}\left(M^{n}\right)$ is a weak homotopy equivalence (a homotopy equivalence if $n=2$ ).

COROLlary 3. The inclusion of the identity component of $P L H\left(M^{n}\right)$ into the identity component of $H\left(M^{n}\right)$ is a weak homotopy equivalence (a homotopy equivalence if $n=2$ ).

We will let $H_{\partial}\left(I^{n}\right)$ denote the set of elements of $H\left(I^{n}\right)$ which equal the identity when restricted to the boundary of $I^{n}$ and $P L H_{\partial}\left(I^{n}\right)=P L H\left(I^{n}\right)$ $\cap H_{\partial}\left(I^{n}\right)$. If $X$ is any space, let $1_{X}$ denote the identity homeomorphism on $X$. If $f \in H(M)$, let $N_{\epsilon}(f)=\{h \in H(M) \mid d(h, f)<\epsilon\}$ where $d$ is a metric on $M$.

2. Proof of Theorem 1. As explained in $\S 1$, the low-dimensional cases are well known.

Lemma 1. If $n>4, P L H_{\partial}\left(I^{n}\right)$ is dense in $H_{\partial}\left(I^{n}\right)$.

Proof. Let $H_{\partial}{ }^{\prime}\left(I^{n}\right)$ be the group of homeomorphisms of $I^{n}$ which fix a neighborhood of $\partial I^{n}$. More precisely: 


$$
\begin{aligned}
& H_{\partial}{ }^{\prime}\left(I^{n}\right)=\left\{h \in H_{\partial}\left(I^{n}\right) \mid \text { for some } 0<a<1,\right. \\
& \qquad h(x)=x \text { whenever } d(x, 0) \geq a\} .
\end{aligned}
$$

By [9, Theorem 3], $H_{\partial}{ }^{\prime}\left(I^{n}\right)$ is a simple subgroup of $H_{\partial}\left(I^{n}\right) . H_{\partial}{ }^{\prime}\left(I^{n}\right)$ is clearly dense in $H_{\partial}\left(I^{n}\right)$. Consider

$$
\begin{aligned}
& H_{\partial}{ }^{\prime \prime}\left(I^{n}\right)=\left\{h \in H_{\partial}{ }^{\prime}\left(I^{n}\right) \mid \text { If } T \text { is a PL structure on } I^{n} \text { and if } \epsilon>0,\right. \\
& \text { there exists } f \in H_{\partial}^{\prime}\left(I^{n}\right) \text { such that } \\
& \qquad f \text { is PL relative to } T \text { and } d(f, h)<\epsilon\} .
\end{aligned}
$$

Claim (following Connell). $H_{\partial}{ }^{\prime \prime}\left(I^{n}\right)$ is normal in $H_{\partial}{ }^{\prime}\left(I^{n}\right)$.

Proof of Claim. Let $h \in H_{\partial}{ }^{\prime \prime}\left(I^{n}\right)$ and $g \in H_{\partial}{ }^{\prime}\left(I^{n}\right)$. We must show $g^{-1} h g$ $\in H_{\partial}{ }^{\prime \prime}\left(I^{n}\right)$. Let $T$ be a PL structure on $I^{n}$ and let $\epsilon>0$ be given. There exists $\delta>0$ such that for all $x, y \in I^{n}, d(x, y)<\delta$ implies $d\left(g^{-1}(x), g^{-1}(y)\right)<\epsilon$. Let $T_{1}$ be the PL structure on $I^{n}$ which is the image of $T$ under $g$. Since $h \in H_{\partial}{ }^{\prime \prime}\left(I^{n}\right)$ there exists $f \in H_{\partial}{ }^{\prime}\left(I^{n}\right)$ which is PL with respect to $T_{1}$ and satisfies $d(h, f)<\delta$. Thus $d\left(g^{-1} h g, g^{-1} f g\right)<\epsilon$. Now $g^{-1} f g$ is PL with respect to $T$. It follows that $g^{-1} h g \in H_{\partial}{ }^{\prime \prime}\left(I^{n}\right)$; the Claim is proved.

Since $H_{\partial}{ }^{\prime}\left(I^{n}\right)$ is dense and simple, it only remains to show that $H_{\partial}{ }^{\prime \prime}\left(I^{n}\right)$ contains some $h_{0} \neq 1_{I^{n}}$. When $n>6$, Connell shows on p. 331 of [7] that any nontrivial symmetric radial expansion of $I^{n}$ which fixes a neighborhood of $\partial I^{n}$ can serve as $h_{0}$. The restriction $n>6$ arises from the radial engulfing lemma used in [7]. But in [3], Bing proves a stronger engulfing lemma which makes Connell's proof valid for $n>4$; see [3, p. 3] and [7, p. 337].

Proof of Theorem 1. Let $H$ be a component of $H(M)$. We show that $H \cap P L H(M)$ is either dense in $H$ or is empty.

We first show the PL homeomorphisms are dense in a neighborhood of $1_{M}$ : i.e., that there is a $\delta>0$ such that whenever $g \in N_{\delta}\left(1_{M}\right)$ and $\epsilon>0$ are given, there exists $h \in P L H(M) \cap N_{\epsilon}(g)$.

Choose an open cover $\left\{B_{1}, \ldots, B_{p}\right\}$ of $M$ so that for each $i, \bar{B}_{i}$ is a PL $n$-ball and so that $D_{i}=\bar{B}_{i} \cap \partial M$ is either empty or a PL $(n-1)$-ball. In [8, p. 19] it is shown that there is a $\delta>0$ so that each $g \in N_{\delta}\left(1_{M}\right)$ can be written as the composition $g=g_{p} g_{p-1} \cdots g_{1}$ of $p$ homeomorphisms such that each $g_{i}$ is supported by $B_{i}$. Since each $g_{i}$ is uniformly continuous, there exist positive numbers $\eta_{1}, \ldots, \eta_{p}$ such that if for each $i$ the homeomorphism $h_{i}$ satisfies $d\left(h_{i}, g_{i}\right)<\eta_{i}$, then $d\left(h_{p} h_{p-1} \cdots h_{1}, g_{p} g_{p-1} \cdots g_{1}\right)<\epsilon$.

If $\bar{B}_{i} \cap \partial M=\varnothing, g_{i} \mid \bar{B}_{i}$ lies in $H_{\partial}\left(\bar{B}_{i}\right)$ and, by the previous lemma, there exists a PL homeomorphism $\tilde{h}_{i} \in H_{\partial}\left(\bar{B}_{i}\right)$ such that $d\left(\tilde{h}_{i}, g_{i} \mid \bar{B}_{i}\right)<\eta_{i}$. Extend $\tilde{h}_{i}$ by the identity to form $h_{i} \in P L H(M)$ and note that $d\left(h_{i}, g_{i}\right)<\eta_{i}$. If $\bar{B}_{i} \cap \partial M=D_{i} \neq \varnothing\left(\right.$ and is therefore a PL $(n-1)$-ball), $g_{i}$ maps $D_{i}$ homeomorphically onto itself and fixes $\partial D_{i}$. By the previous lemma there is a PL homeomorphism $f_{i} \in H_{\partial}\left(D_{i}\right)$ approximating $g_{i} \mid D_{i}$. Extending $f_{i}$ by the identity we get a PL homeomorphism $\tilde{f}_{i}: \partial \bar{B}_{i} \rightarrow \partial \bar{B}_{i}$ which approximates $g_{i} \mid \partial \bar{B}_{i}$. Thus $\tilde{f}_{i} g_{i}^{-1} \mid \partial \bar{B}_{i}$ approximates $1_{\partial \bar{B}_{i}}$. Coning at a point in the interior of $B_{i}$, we obtain a homeomorphism $F_{i}$ of $B_{i}$ which agrees with $\tilde{f}_{i} g_{i}^{-1}$ on $\partial \bar{B}_{i}$ and which approximates $1_{B_{i}}$. Let $g_{i}{ }^{\prime}=F_{i} g_{i}: \bar{B}_{i} \rightarrow \bar{B}_{i}$. Note that $g_{i}{ }^{\prime}$ approximates $g_{i}$ and is PL on $\partial \bar{B}_{i}$. Omitting epsilonics, we may say that $d\left(g_{i}{ }^{\prime}, g_{i} \mid B_{i}\right)<\eta_{i} / 2$. Note 
that $g_{i}{ }^{\prime}$ fixes $\partial \bar{B}_{i} \backslash$ int $D_{i}$. By coning, construct a PL homeomorphism $g_{i}{ }^{\prime \prime}: \bar{B}_{i}$ $\rightarrow \bar{B}_{i}$ which extends $g_{i}{ }^{\prime} \mid \partial B_{i}$. Then $g_{i}{ }^{\prime}\left(g_{i}{ }^{\prime \prime}\right)^{-1} \in H_{\partial}\left(B_{i}\right)$, so there exists $\tilde{g}_{i}$ $\in P L H_{\partial}\left(B_{i}\right)$ such that $d\left(\tilde{g}_{i}, g_{i}{ }^{\prime}\left(g_{i}{ }^{\prime \prime}\right)^{-1}\right)<\eta_{i} / 2$. Extend $\tilde{g}_{i} g_{i}{ }^{\prime \prime}$ by the identity to $h_{i} \in P L H(M)$ and note that $d\left(h_{i}, g_{i}\right)<\eta_{i}$. The required $h$ is $h_{p} \cdots h_{1}$.

Now let $H$ be a component of $H(M)$ such that $H \cap P L H(M) \neq \varnothing$. Let $A=\mathrm{cl}_{H}(H \cap P L H(M))$. We will show that $A$ is open in $H$, from which it will follow that $A=H$. Let $f \in A$. Let $\delta$ be as in the first part of the proof, and assume without loss of generality that $N_{2 \delta}(f) \subset H$. The local connectedness of $H(M)$ is used here. Let $g_{0} \in N_{\delta}(f)$. We must show that $g_{0} \in A$. Let $\eta>0$ be given: we may assume $\eta \leq \delta$. Since $d\left(g_{0} f^{-1}, 1_{M}\right)<\delta$, there exists $h \in P L H(M)$ such that $d\left(h, g_{0} f^{-1}\right)<\eta / 2$ and hence $d\left(h f, g_{0}\right)<\eta / 2$. Since $h$ is uniformly continuous, there exists $\gamma>0$ such that $d(h(x), h(y))<\eta / 2$ whenever $d(x, y)<\gamma$. Choose $f^{\prime} \in P L H(M)$ such that $d\left(f^{\prime}, f\right)<\gamma$. Then $h f^{\prime} \in P L H(M)$ and $d\left(h f^{\prime}, g_{0}\right) \leq d\left(h f^{\prime}, h f\right)+d\left(h f, g_{0}\right)<\eta$. Therefore $h f^{\prime}$ $\in N_{\eta}\left(g_{0}\right) \cap H$. Since $h f^{\prime}$ is PL, $g_{0} \in A$.

3. Proof of Theorem 2. A subset $X^{\prime}$ of a metric space $X$ is said to have the finite-dimensional compact absorption property (f.d. cap) if $X^{\prime}=\cup_{n=1}^{\infty} A_{n}$ where (i) each $A_{n}$ is finite dimensional and compact, and (ii) given a finitedimensional compactum $A$, a closed subset of $B$ of $A$, an embedding $f: A \rightarrow X$ such that $f(B) \subset X^{\prime}$, and a number $\epsilon>0$, there exists an embedding $h: A \rightarrow X$ such that $d(f, h)<\epsilon, h(A) \subset X^{\prime}$ and $h(b)=f(b)$ whenever $b$ $\in B$.

This property characterizes $\left(l_{2}, l_{2}\right)$-manifold pairs: precisely

Proposition 1 (West [21]). Let $\left(X, X^{\prime}\right)$ be a pair of metric spaces such that $X^{\prime}$ has the f.d. cap in $X$. Then $\left(X, X^{\prime}\right)$ is an $\left(l_{2}, l_{2}\right)$-manifold pair if and only if $X$ is an $l_{2}$-manifold.

Where there is no confusion, we will not distinguish between a complex $P$, and its underlying point set, $|P|$. Let $\overline{P L H}(M)$ be the closure of $P L H(M)$ in $H(M)$.

Lemma 2. Let $P$ be a finite complex and $B$ a closed subset of $P$. Suppose $f: P \rightarrow \overline{P L H}(M)$ is a map such that $f(B) \subset P L H(M)$ and $f(P \backslash B) \cap f(B)$ $=\varnothing$. Then given $\epsilon>0$, there is a map $g: P \rightarrow P L H(M)$ such that $d(f, g)<\epsilon$, $g|B=f| B$ and $g(P \backslash B) \cap g(B)=\varnothing$.

Proof. Let $n=\operatorname{dim} P$. Whenever $W$ is a closed subset of $P \backslash B$ let

$$
\eta_{W}=1 / 4 \min (\epsilon, d(f(W), f(B))) .
$$

Using the fact that $P L H(M)$ is uniformly locally contractible, form a triangulation $T$ of $P \backslash B$ satisfying:

(a) for each simplex $\sigma$ of $T$ there is a sequence $0<\delta_{\sigma, 0}<\delta_{\sigma, 1}<\cdots<\delta_{\sigma, n}$ $\leq \eta_{\sigma}$ such that any subset of $P L H(M)$ of diameter $<3 \delta_{\sigma, i}$ is contractible in a subset of $P L H(M)$ of diameter $<\delta_{\sigma, i+1}, 0 \leq i \leq n-1$; and

(b) $\operatorname{diam} f(\sigma)<\delta_{\sigma, 0}$. (First choose a triangulation $\tilde{T}$, next choose the $\delta_{\sigma, i}$ 's with reference to $\tilde{T}$ to satisfy (a); then subdivide $\tilde{T}$ to get $T$ satisfying (b).)

Define $g: T^{0} \rightarrow P L H(M)$ as follows. If $v$ is a vertex of $T^{0}$, let $g(v)$ be any 
point of $P L H(M)$ such that $d(g(v), f(v))<\delta_{\sigma, 0}$ for each of the (finite number of) simplexes $\sigma$ of $T$ which contains $v$ as a vertex.

We next define $g: T^{1} \rightarrow P L H(M)$. Let $\tau$ be a 1-simplex of $T^{1}$ and let $\sigma_{0}<T$ be a simplex of which $\tau$ is a face. Then $\operatorname{diam} f(\tau) \leq \operatorname{diam} f\left(\sigma_{0}\right)$. Hence $\operatorname{diam} g(\partial \tau)<3 \delta_{\sigma_{0}, 0}$. We can therefore define

$$
g \mid \tau: \tau \rightarrow P L H(M)
$$

extending $g \mid \partial \tau$ in such a way that diam $g(\tau)<\delta_{\sigma, 1}$ for each of the (finite number of) simplexes $\sigma$ of $T$ which contains $\tau$ as a face.

Assume, inductively, that we have defined $g \mid T^{j}: T^{J} \rightarrow P L H(M)$, such that if $\tau$ is a $j$-simplex of $T$, then $\operatorname{diam} g(\tau)<\delta_{\sigma, j}$ for each of the (finite number of) simplexes $\sigma$ of $T$ which contains $\tau$ as a face.

Let $\tau$ be a $(j+1)$-simplex of $T$. Note that $\operatorname{diam} g(\partial \tau)<3 \delta_{\sigma, j}$ for each simplex $\sigma$ of $T$ which contains $\tau$ as a face. Therefore $g \mid \partial \tau$ can be extended to $\tau$ in such a way that $\operatorname{diam} g(\tau)<\delta_{\sigma, j+1}$ for each simplex $\sigma$ of $T$ of which $\tau$ is a face.

Thus, by induction we define a continuous map $g: T \rightarrow P L H(M)$ so that if $\tau$ is a simplex of $T, \operatorname{diam} g(\tau)<\eta_{\tau}$. Finally define $g$ on $B$ to agree with $f$.

We now show that $d(f, g)<\epsilon$. If $p \in B, d(f(p), g(p))=0$. If $p \in P \backslash B$ let $\tau$ be a simplex of $T$ containing $p: d(f(p), g(p)) \leq 3 \eta_{\tau}<\epsilon$.

To show that $g(P \backslash B) \cap g(B)=\varnothing$, we observe that if $p \in P \backslash B$ and $p$ lies in the simplex $\tau$ of $T$,

$$
\begin{aligned}
d(g(p), g(B)) & \geq d(f(p), f(B))-d(f(p), g(p)) \\
& \geq d(f(p), f(B))-3 \eta_{\tau}>0 .
\end{aligned}
$$

Finally we show that $g$ is continuous on $B$. Let $g \in B$ and $\eta>0$ be given. Choose $\delta>0$ so that if $d(p, q)<\delta, d(f(p), f(q))<\eta / 2$. Assume $p \in P \backslash B$. Then

$$
\begin{aligned}
d(g(p), g(q)) & \leq d(g(p), f(p))+d(f(p), f(q)) \\
& \leq d(f(\tau), f(B))+d(f(p), f(q)) \leq 2 d(f(p), f(q))<\eta .
\end{aligned}
$$

Let $s$ denote the countable infinite product of open unit intervals and $s_{f}=\left\{\left\{x_{i}\right\} \in s \mid\right.$ for at most finitely many $\left.i, x_{i} \neq 0\right\}$ with metric $d(x, y)$ $=\sum_{i} \frac{1}{2} i\left|x_{i}-y_{i}\right|$.

Lemma 3. Let $P$ be a finite complex, $B$ a closed subset of $P, V$ an open subset of $s_{f}$. Let $g: P \rightarrow V$ be a continuous map. Then given $\epsilon>0$, there is a map $h: P \rightarrow V$ such that $h|B=g| B, d(g, h)<\epsilon, h(B) \cap h(P \backslash B)=\varnothing$ and $h \mid P \backslash B$ is injective.

Proof. This is standard infinite-dimensional topology: we sketch the proof, leaving epsilonics to the reader. Let $p_{m}: s_{f} \rightarrow s_{f}$ be the projection onto the first $m$ coordinates. If $m$ is large, $g$ is homotopic in $V$ to $p_{m} g$ by a small homotopy. If $m$ is large enough, $p_{m} g$ is homotopic in $V$ to an embedding $h^{\prime}: P \rightarrow V$ by a small homotopy. $h^{\prime} g^{-1} \mid g(B)$ is a homeomorphism of $g(B)$ onto $h^{\prime}(B)$ which is homotopic to the identity by a small homotopy. By Theorem 2.25 of [5], there 
is a homeomorphism $k$ of $s_{f}$, close to the identity, extending $h^{\prime} g^{-1} \mid g(B)$. The required $h$ is $k^{-1} h^{\prime}: P \rightarrow V$.

Proof of Theorem 2. By Theorem $1, H^{*}(M)$ is an $l_{2}$-manifold if and only if $H(M)$ is. By Proposition 1 it is enough to show that $P L H(M)$ has the f.d. cap in $H^{*}(M)$. By [12, Theorem 1.9], $P L H(M)$ is the countable union of finite-dimensional compacta. We must verify (ii) the f.d. cap.

Suppose we are given a finite-dimensional compactum $A$, a compact subset $B$, an embedding $f: A \rightarrow H^{*}(M)$ such that $f(B) \subset P L H(M)$, and $\epsilon>0$. By Theorem 1, $\overline{P L H}(M)=H^{*}(M)$. Since $H^{*}(M)$ is locally contractible, $f$ extends to a map $f^{\prime}: P \rightarrow H^{*}(M)$ where $P$ is a finite complex (see [15, p.150]). As explained in $\S 1, P L H(M)$ is an $l f$-manifold. By Theorem 7 of [14], $P L H(M)$ is homeomorphic to an open subset of $l f$, and hence to an open subset of $s_{f}$ since $l f$ and $s_{f}$ are homeomorphic [1], [2]. Thus, Lemmas 2 and 3 give a map $h: P \rightarrow P L H(M)$ with $d\left(f^{\prime}, h\right)<\epsilon, h\left|B^{\prime}=f^{\prime}\right| B^{\prime}$ where $B^{\prime}$ $=f^{\prime-1}(f(B)), h\left(B^{\prime}\right) \cap h\left(P \backslash B^{\prime}\right)=\varnothing$ and $h \mid P \backslash B^{\prime}$ is injective. $h \mid A$ is the desired embedding.

\section{REFERENCES}

1. R. D. Anderson, On sigma-compact subsets of infinite-dimensional spaces, (preprint).

2. C. Bessaga and A. Pełczyński, The estimated extension theorem, homogeneous collections and skeletons, and their applications to the topological classification of linear metric spaces, Fund. Math. 69 (1970), 153-190. MR 42 \#8227.

3. R. H. Bing, Radial engulfing, Conference on the Topology of Manifolds (Michigan State Univ., E. Lansing, Mich., 1967), Prindle, Weber \& Schmidt, Boston, Mass., 1968, pp. 1-18. MR 38 \#6560.

4. - Locally tame sets are tame, Ann. of Math. (2) 59 (1954), 145-158. MR 15, 816.

5. T. A. Chapman, Dense sigma-compact subsets of infinite-dimensional manifolds, Trans. Amer. Math. Soc. 154 (1971), 339-426. MR 44 \# 1058.

6. A. V. Cernavskii, Local contractibility of the homeomorphism group of a manifold, Dokl. Akad. Nauk SSSR 182 (1968), 510-513 = Soviet Math. Dokl. 9(1968), 1171-1174. MR 38 \# 5241.

7. E. H. Connell, Approximating stable homeomorphisms by piecewise linear ones, Ann. of Math. (2) 78 (1963), 326-338. MR 27 \#4238.

8. R. D. Edwards and R. C. Kirby, Deformations of spaces of embeddings, Ann. of Math. (2) 93 (1971), 63-88. MR 44 \#1032.

9. G. M. Fisher, On the group of all homeomorphisms on a manifold, Trans. Amer. Math. Soc. 97 (1960), 193-212. MR 22 \#8487.

10. D. Gauld, Local contractibility of $P L(M)$ for a compact manifold, Preprint, Univ. of Auckland Dept. of Math. Rep. Ser., no. 47, 1973.

11. R. Geoghegan, On spaces of homeomorphisms, embeddings, and functions. I, Topology 11 (1972), 159-177. MR 45 \# 4349.

12. Un spaces of homeomorphisms, embeddings, and functions. II. The piecewise linear case, Proc. London Math. Soc. (3) 27 (1973), 463-483.

13. W. E. Haver, Locally contractible spaces that are absolute neighborhood retracts, Proc. Amer. Math. Soc. 40 (1973), 280-284.

14. D. W. Henderson and J. E. West, Triangulated infinite-dimensional manifolds, Bull. Amer. Math. Soc. 76 (1970), 655-660. MR 41 \#2714; erratum, 41 p. 1966.

15. S. T. Hu, Theory of retracts, Wayne State Univ. Press, Detroit, Mich., 1965. MR 31 \#6202.

16. J. Keesling and D. Wilson, The group of PL-homeomorphisms of a compact PL-manifold is an $l_{2}^{f}$-manifold, Trans. Amer. Math. Soc. 193 (1974), 249-256.

17. R. Kirby, Lectures on triangulations of manifolds, Mimeographed Notes, Univ. of California, Los Angeles, Calif., 1969.

18. R. Luke and W. K. Mason, The space of homeomorphisms on a compact two-manifold is an 
absolute neighborhood retract, Trans. Amer. Math. Soc. 164 (1972), 273-285. MR 46 \# 849.

19. T. Rádo, Über den Begriff Riemannschen Fläche, Szeged Univ. Act. 2 (1925), 101-121.

20. H. Toruńczyk, Absolute retracts as factors of normed linear spaces, Fund. Math. (to appear).

21. J. E. West, The ambient homeomorphy of an incomplete subspace of infinite-dimensional Hilbert spaces, Pacific J. Math. 34 (1970), 257-267. MR 43 \#2748.

Department of Mathematical Sciences, State University of New York at Binghamton, BinghamTon, NeW YoRK 13901

Department of Mathematics, University of Tennessee, Knoxville, Tennessee 37916 